\title{
Comparison of Randomized Solutions for Constrained Vehicle Routing Problem
}

\author{
İbrahim Ethem Demirci \\ Department of Mathematics, \\ Hacettepe University \\ Ankara, Turkey \\ demirciibrahim.e@gmail.com
}

\author{
Şaziye Ece Özdemir \\ Department of Mathematics, \\ Pamukkale University, \\ Denizli, Turkey and \\ Department of Mathematics, \\ Hacettepe University, \\ Ankara, Turkey \\ saziyeo@pau.edu.tr
}

\author{
Oğuz Yayla \\ Department of Mathematics, \\ Hacettepe University, \\ Ankara, Turkey \\ oguz.yayla@ hacettepe.edu.tr
}

\begin{abstract}
In this short paper, we study capacity constrained vehicle routing problem (CVRP) and its solution by randomized Monte Carlo methods. For solving CVRP we use some pseudorandom number generators commonly used in practice. We use linear, multiple-recursive, inversive and explicit inversive congruential generators and obtain random numbers from each to provide a route for CVRP. Then we compare the performance of pseudorandom number generators with respect to the total time the random route takes. We also constructed an open-source library github.com/iedmrc/binary-cws-mes on solving CVRP by Monte-Carlo based heuristic methods.

Index Terms - constrained vehicle routing problem, pseudoran-
\end{abstract} dom number generators, Monte Carlo simulation.

\section{INTRODUCTION}

The Monte Carlo method is used in computational algorithms where repeated random sampling is needed. It provides randomness to solve problems that might be deterministic in principle. Many of the most useful techniques use deterministic pseudorandom sequences because it is simple to test and re-run the simulation. Monte Carlo method has a lot of areas of application, for example, physical science, engineering, computational biology, applied statistics, computer graphics, finance, business and mathematics [17].

Monte Carlo method uses random samples; hence needs a good random number generation. There are two main types of random number generation: true random generation and pseudorandom generation. We use a pseudorandom generator because of its deterministic behaviour, that is, one can re-run the generation process.

There are various pseudorandom generators in literature. In this study, we examine some of them: linear congruential method (LCG), inversive congruential generator (ICG), Lehmer congruential generation (LehmerCG) and explicit inversive congruential generator (EICG). Then we test their performance on the capacitated vehicle routing problem (CVRP) on TSPLIB data sets [16].

\section{RELATED WORK}

The vehicle routing problem (VRP) was first studied in [8] by George Dantzig and John Ramser in 1959, where petrol deliveries were considered algorithmically. The ultimate aim of the VRP is to minimize time, distance or budget cost of route. For other variants of VRP, see the recent study [22].

Several methods have been used for solving CVRP. There are heuristic and meta-heuristic methods. Heuristic methods speed up duration for reaching a reasonable and optimal solution, and they are based on four principles: understand the problem, make a plan, apply the plan, evaluate and adjust respectively. For example; Clark \& Wright's Savings [6] is used in heuristic methods thoroughly. See also its generalizations in [2], [9], [18] and recent variants in [3], [19].

In 1964, Clarke and Wright developed a saving algorithm based on Dantzig and Ramser's greedy approximation, which is one of the most known heuristic solution methods for VRP. This is called as CWS algorithm. In this study we consider Capacitated VRP (CVRP), which is like VRP with the additional constrain that every vehicle has a capacity of the amount that it can carry.

There are some studies on applying the Monte Carlo method to the VRP and CVRP. For VRP it was applied the first time in the 1970s and random sampling for the distance constrained VRP applied in [7]. Authors in [21] use the Monte Carlo method in the CWS algorithm to provide a better solution for VRP. They also applied the Monte Carlo method for CVRP. In this paper we mainly based on the methods given in [21] and their improvements. There are other studies combine MonteCarlo simulation and heuristic method for CVRP, see [4], [5], [10], [11].

On the other hand, metaheuristic methods has made great progress in the past 15 years for VRP and classified as local search, population search, and learning algorithms, which were used much earlier than CVRP [12].

Another randomized methodology applied in solving VRP is GRASP (greedy randomized adaptive search procedure). It is a recursive metaheuristic and each recursive compose two stages that are construction and local search. It was for the first time exploited by Feo and Resende in 1989 and largely used in multiple applications [1]. GRASP solutions might be generated in the same number of time need for the local search 
procedure to converge from a single random start.

\section{Methodology}

The goal of this section is to describe the methodology we follow to provide a randomized solution for CVRP. Initially, there are a lot of pseudorandom numbers generation methods, see [13] and [14]. In this paper we consider only inversive congruential generator (ICG), multiple-recursive generator (MRG), Lehmer congruential generator (LehmerCG), linear congruential generator (LCG). The main aim of this paper is to compare the CVRP solutions obtained by using these pseudorandom generation methods.

The first pseudorandom number generation method is Lehmer congruential generator (LehmerCG), also known as Park Miller random number generator, the general formula is $x_{k+1}=a \cdot x_{k} \bmod m$ where $m$ is a prime number or power of a prime number, $a$ is the multiplier and the seed $x_{0}$ is expected to be coprime to $m$.

The linear congruential generator (LCG), introduced in 1958 by W.E Thomson and A Rotenberg, is an extension of the Lehmer generator and it is the most used and studied generator. This method is still practically used since it is so simple to define. It has only two parameters namely a large integer $m$ and integers $a, b$ with $\operatorname{gcd}(a, m)=1$. Let $\mathbb{Z}_{m}=\{0,1,2, \ldots m-1\}$ and choose an initial value $x_{0} \in \mathbb{Z}_{m}$ such that $\operatorname{gcd}\left(x_{0}, m\right)=1$. Then we generate a sequence $x_{n+1}=a \cdot x_{n}+b \bmod m$ for $n=0,1, \ldots$ where $m$ is referred to as the modulus and $a$ is the multiplier.

The third random number generation is multi-recursive generator (MRG). It generates a random sequence $x_{n}$ as $x_{n+1}=a \cdot x_{n}+b \cdot x_{n-1} \bmod m$ for $n=1, \ldots$ and initial values $x_{0}, x_{1} \in \mathbb{Z}_{m}$ where $m$ is referred to as the modulus and $a, b$ are multipliers.

The fourth random number generation is the inversive congruential generator (ICG), which is a nonlinear generator introduced by Eichenauer and Lehn in 1986. In this method, $p$ is a prime number, $x^{-1} \cdot x=1 \bmod p$ where $x^{-1}$ is called the inverse of an integer $x$ and we generate random numbers with the standard formula for a chosen seed $x_{0}$

$$
\begin{gathered}
x_{i+1}=\left(a \cdot x_{i}^{-1}+c\right) \bmod p \quad \text { if } \quad x_{i} \neq 0 \\
x_{i+1}=c \text { if } x_{i}=0 .
\end{gathered}
$$

Lastly, the explicit inversive congruential generator (EICG) was introduced by Eichenauer-Herrmann in 1993 and it is a different form of inversive generator. It is defined as $x_{k}=$ $\overline{k+k_{0}} \bmod p$ where $k_{0}$ is a seed value.

We use pseudorandom numbers at various algorithms such as Nearest Neighbor Insertion (NNI), Clarke \& Wright's Savings Algorithm (CWS), Monte Carlo Simulation.

Below, we consider that we have $m$ vehicles with equal capacity $Q$ and they are assigned to $n$ jobs with demands $d_{i}$, $i=1,2, \ldots, n$.

\section{A. Nearest Neighbor Insertion}

The Nearest Neighbor Insertion (NNI) Algorithm is one of the methods for the vehicle routing problem. The NNI algorithm firstly selects a vehicle and a customer randomly then adds another customer which is the nearest unvisited until the vehicle's capacity gets full. After that, we choose another vehicle, and similar steps are applied until all customers are visited, or all vehicles' capacity is full, which depends on tightness. Tightness is a relation between all customers' demands and the total capacity of the vehicle, and defined as

$$
T=\frac{\sum d_{i}}{Q m} \text {. }
$$

If the $\mathrm{T}$ is very close to 1 , the algorithm most likely does not yield a proper result. We present the pseudocode of the NNI method in Algorithm 1.

Example 1: Let us examine the NNI method with an example where we have three vehicle $(m=3)$ with capacities $Q=8$, there are 8 customers $j_{i}(i=1 \ldots 8)$ with demands $d_{1}=2, d_{2}=4, d_{3}=3, d_{4}=1, d_{5}=5, d_{6}=2, d_{7}=$ $2, d_{8}=3$ respectively. In Table I, we give the distance between each customer. Firstly a customer $\left(j_{1}\right)$ is selected randomly,

TABLE I

DisTANCE MATRIX

\begin{tabular}{|l|l|l|l|l|l|l|l|l|}
\hline$j_{i}$ & $j_{1}$ & $j_{2}$ & $j_{3}$ & $j_{4}$ & $j_{5}$ & $j_{6}$ & $j_{7}$ & $j_{8}$ \\
\hline$j_{1}$ & 0 & 10 & 20 & 13 & 12 & 7 & 15 & 9 \\
\hline$j_{2}$ & 10 & 0 & 4 & 7 & 6 & 20 & 13 & 17 \\
\hline$j_{3}$ & 20 & 4 & 0 & 11 & 6 & 19 & 14 & 8 \\
\hline$j_{4}$ & 13 & 7 & 11 & 0 & 3 & 5 & 6 & 18 \\
\hline$j_{5}$ & 12 & 6 & 6 & 3 & 0 & 9 & 12 & 16 \\
\hline$j_{6}$ & 7 & 20 & 19 & 5 & 9 & 0 & 15 & 11 \\
\hline$j_{7}$ & 15 & 13 & 14 & 6 & 12 & 15 & 0 & 21 \\
\hline$j_{8}$ & 9 & 17 & 8 & 18 & 16 & 11 & 21 & 0 \\
\hline
\end{tabular}

and NNI method chooses the nearest unvisited customer $\left(j_{6}\right)$, then checks whether the sum of their demands exceeds the capacity of the vehicle $(2+2 \leq 8)$. If enough capacity exists then the method constitutes a route as $\left[\begin{array}{ll}j_{1} & j_{6}\end{array}\right]$. If this is continued by selecting the nearest unvisited customer to $j_{6}$, route $\left[\begin{array}{lll}j_{1} & j_{6} & j_{4}\end{array}\right]$ is obtained for the first vehicle, if we add to $j_{5}$ for the nearest customer to $j_{4}$, this will surpass the capacity. Therefore pick $j_{5}$ for the second vehicle randomly and the one closest to it $j_{2}$ or $j_{3}$ but we prefer to $j_{3}$ because $j_{2}$ surpasses the capacity of the second vehicle thus the route is occurred as $\left[\begin{array}{ll}j_{5} & j_{3}\end{array}\right]$. Lastly take the third vehicle and pick one of the remaining jobs randomly $j_{2}$ and one of the nearest to it $j_{7}$ in the rest of jobs. Finally, the latest customer $j_{8}$ and its demand is suitable for the vehicle's capacity, so the route constituted for the third vehicle like $\left[\begin{array}{lll}j_{2} & j_{7} & j_{8}\end{array}\right]$.

\section{B. Clark \& Wright's Savings (CWS) Algorithm}

Clark and Wright [6] have published an algorithm for the vehicle routing problem based upon saving opinion, which is a heuristic algorithm, so the solution may not be provided with an optimal solution. In CWS algorithm, we have an $(n+1) \times(n+1)$ distance matrix $C$ whose entries $c_{i j}$ are the distance between two customers $i$ and $j$ for $i, j=0,1,2, \ldots, n$, 


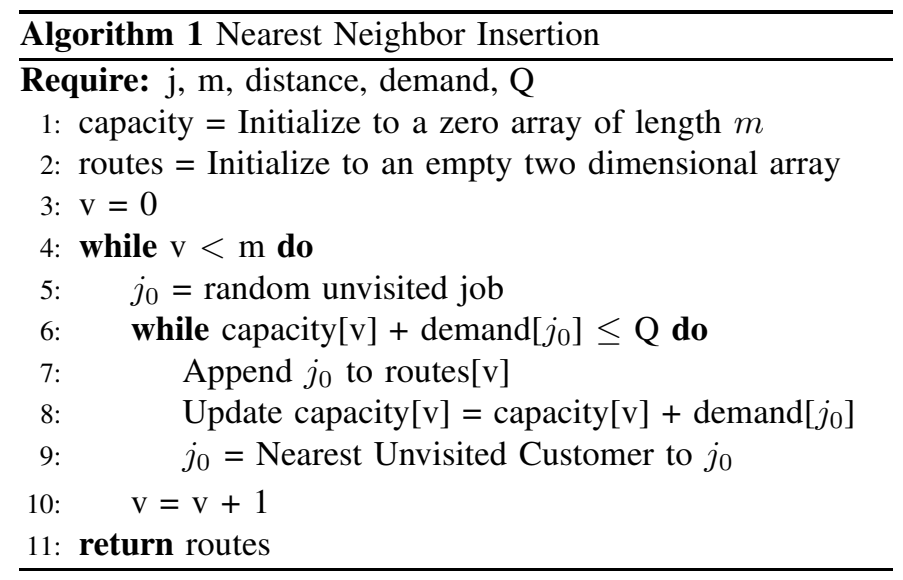

TABLE II

Customer Demand

\begin{tabular}{|l|l|}
\hline customer & demand \\
\hline$j_{1}$ & 2 \\
\hline$j_{2}$ & 4 \\
\hline$j_{3}$ & 3 \\
\hline$j_{4}$ & 1 \\
\hline$j_{5}$ & 5 \\
\hline$j_{6}$ & 2 \\
\hline$j_{7}$ & 2 \\
\hline$j_{8}$ & 1 \\
\hline
\end{tabular}

where we set the index 0 to the starting point of the vehicles, say depot. Then, the saving list $s_{i j}$ is created by using the following formula

$$
s_{i j}=c_{0 i}+c_{0 j}-c_{i j}
$$

for $i, j=1,2, \ldots, n$. The steps of the CWS method are given in Algorithm 2 where the second part between steps 6 and 12 is called the process procedure of $(\mathrm{i}, \mathrm{j})$ pair, and this process part is used in the next section. Let us examine this algorithm with an example.

Example 2: We have three vehicles $(\mathrm{m}=3)$ with capacity 10 $(\mathrm{Q}=10)$ and there are eight jobs $(\mathrm{j}=8)$ with demands given in Table II] If we take into account saving matrix, constitutes saving list from the high savings pair to the lowest saving pair such that 1-2, 2-4, 1-3, 2-3, 3-4, 3-6, 2-6, 2-7, 2-8, 1-4, 3-5, $4-6,4-8,6-8,4-5,1-6,3-8,2-5,1-5,5-6,7-8,4-7,6-7,1-7$ respectively. First, we choose to the highest saving pairs 12 and 2-4, then check whether the sum of their demands to

TABLE III

DisTANCE MATRIX

\begin{tabular}{|l|l|l|l|l|l|l|l|l|l|}
\hline$c_{i j}$ & $j_{0}$ & $j_{1}$ & $j_{2}$ & $j_{3}$ & $j_{4}$ & $j_{5}$ & $j_{6}$ & $j_{7}$ & $j_{8}$ \\
\hline$j_{0}$ & 0 & 10 & 20 & 13 & 12 & 7 & 15 & 9 & 10 \\
\hline$j_{1}$ & 10 & 0 & 4 & 7 & 6 & 20 & 13 & 17 & 9 \\
\hline$j_{2}$ & 20 & 4 & 0 & 11 & 6 & 19 & 14 & 8 & 13 \\
\hline$j_{3}$ & 13 & 7 & 11 & 0 & 3 & 5 & 6 & 18 & 14 \\
\hline$j_{4}$ & 12 & 6 & 6 & 3 & 0 & 9 & 12 & 16 & 8 \\
\hline$j_{5}$ & 7 & 10 & 19 & 5 & 9 & 0 & 15 & 11 & 9 \\
\hline$j_{6}$ & 15 & 13 & 14 & 6 & 12 & 15 & 0 & 21 & 13 \\
\hline$j_{7}$ & 9 & 17 & 9 & 18 & 16 & 11 & 21 & 0 & 13 \\
\hline$j_{8}$ & 10 & 9 & 13 & 14 & 8 & 9 & 11 & 13 & 0 \\
\hline
\end{tabular}

TABLE IV

SYMMETRIC SAVING MATRIX

\begin{tabular}{|l|l|l|l|l|l|l|l|l|}
\hline$s_{i j}$ & $j_{1}$ & $j_{2}$ & $j_{3}$ & $j_{4}$ & $j_{5}$ & $j_{6}$ & $j_{7}$ & $j_{8}$ \\
\hline$j_{1}$ & 0 & 26 & 23 & 16 & 7 & 8 & 2 & 13 \\
\hline$j_{2}$ & & 0 & 22 & 26 & 8 & 21 & 20 & 17 \\
\hline$j_{3}$ & & & 0 & 22 & 15 & 22 & 4 & 9 \\
\hline$j_{4}$ & & & & 0 & 10 & 15 & 5 & 14 \\
\hline$j_{5}$ & & & & & 0 & 7 & 5 & 8 \\
\hline$j_{6}$ & & & & & & 0 & 3 & 14 \\
\hline$j_{7}$ & & & & & & & 0 & 6 \\
\hline$j_{8}$ & & & & & & & & 0 \\
\hline
\end{tabular}

surpass the capacity of a vehicle or not, $(2+4+1 \leq 10)$, if not, 1-2-4 will be added to one route, then 1-3 is added to a route because it does not exceed total capacity of the first vehicle. Therefore a route is created for the first vehicle such that 3-1-2-4. We can skip the pairs which are selected previously because no need to include them in another route. For the second vehicle, we first pick the highest saving 6-8. Next, we choose the pair 5-6 and add it to the route as 5-6-8 as total demand $(2+1+5 \leq 10)$ ensures the second vehicle's capacity. The next pair is $7-8$ but we cannot add to the route because it exceeds the total capacity. Thus the last vehicle will go to the last customer 7 only.

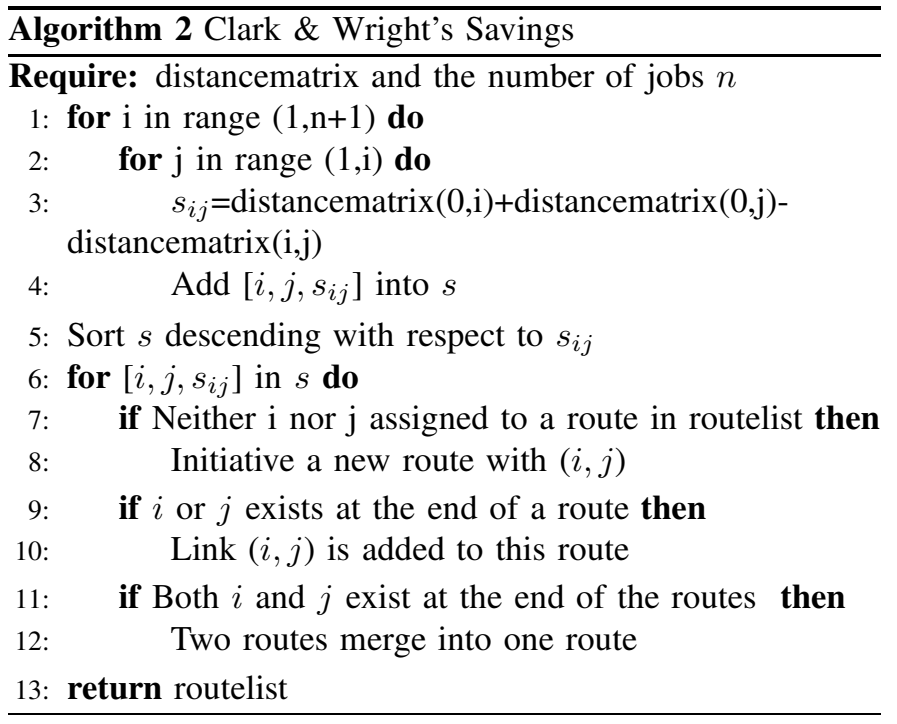

\section{Monte Carlo Simulation}

The Monte Carlo Simulation is used in the heuristic methods. Firstly one chooses a root node from the search space. We apply $r$ random simulation to its children nodes, by going from the current node until a leaf node. This algorithm visits $n r$ nodes at end of the each step, and so has the highest score or highest average out of $r$ probes opt for as a "best child" and then the best child is used for expansion.

\section{MCS-NNI Method}

This method is used with a probability function as described in the following equation, instead of selecting the nearest unvisited customer. 


$$
p_{v_{i}}=1-\frac{f\left(v_{i}\right)}{\bar{n}}
$$

where $f\left(v_{i}\right) \in[\bar{n}, 1]$ indicates the rank of customers such that the nearest customer has rank 1, another nearest customer's rank 2, etc. The step is used for making selections (it can be thought of as selecting $\mathrm{n}$ children) and then $r$ simulations applied to each of $\mathrm{n}$ children as a Monte Carlo Simulation. On the other hand, there is not much information about the NNI method because this method is outperformed by the CWS method in the literature.

\section{E. Binary-CWS and Binary-CWS-MCS Method}

This method uses Monte Carlo Simulation and Clarke \& Wright's savings list. For each method, the saving list is ordered from top to bottom. Then generated random numbers are used for the election of the current saving pair. In the BinaryCWS method, whose pseudocode is presented in Algorithm 3, we pick a $p$ probability value randomly in $0.05 \leq p \leq 0.40$ as mentioned in [21]. The most crucial point is that if $p$ value is very close to 0 , the algorithm gives solution almost equal to CWS algorithm or larger value of $p$ will end up with chaos. Thus, we choose $p$ value such that $0.05 \leq p \leq 0.40$.

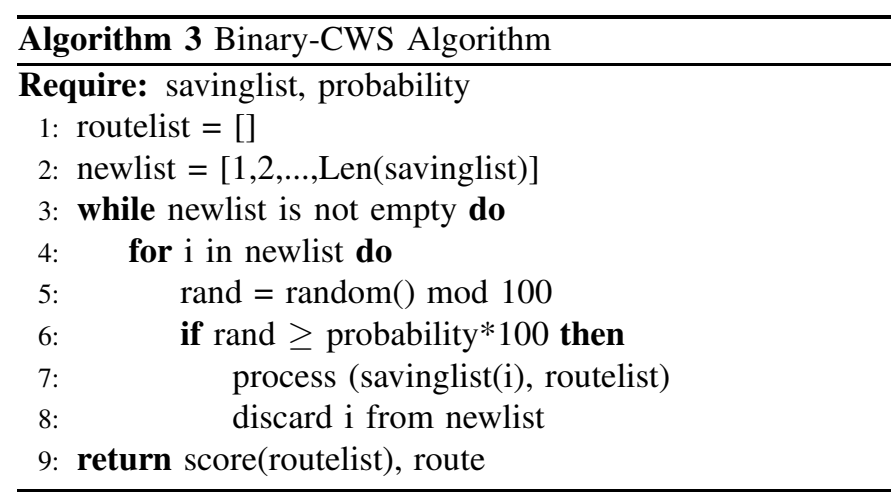

In the Binary-CWS method, we need saving-list from top to bottom and a probability value $p$ between $0.05 \leq p \leq 0.40$. We first pick a pair for the first vehicle, which has the highest score in the saving-list. We note that we use the parallel version method in the CWS algorithm and create routes for many vehicles at the same time. We generate random numbers. Then we compare $p$ value with the chosen random number $(\bmod 100)$, whether to continue with the next pair or not. If random number $(\bmod 100) \geq 100 p$, then we choose the next highest-scoring pair. When the demand is smaller than capacity, the pair is added to the route. Otherwise this job is assigned to another suitable job. For the next steps of the algorithm we scan the saving-list, because we do not choose pair even it contains one of the common job previously selected pairs. By repeating all these steps, a route is created for each vehicle. Let us explain with an example.

Example 3: There are 4 vehicles $(\mathrm{m}=4), 12$ jobs $(\mathrm{n}=12)$ also customers' demands are $\left(d_{1}=1200, d_{2}=1700, d_{3}=1500\right.$, $d_{4}=1400, d_{5}=1700, d_{6}=1400, d_{7}=1200, d_{8}=1900, d_{9}=1800$, $\left.d_{10}=1600, d_{11}=1700, d_{12}=1100\right)$ respectively and distance matrix given in Table V. Assume that vehicles have equal capacity $(\mathrm{Q}=6000)$. We compute the saving list $7-5,10-5$, $8-5,10-7,7-4,11-5,5-3,5-4,5-2,7-2,7-3,12-7,11-10$, $12-5,11-7,8-7,11-8,9-7,11-4,10-6,7-6,10-8,12-10,10-$ $9,11-3,11-9,10-4,9-5,12-9,12-4,12-11,12-3,11-2,9-8$, $8-6, \ldots$ On the other hand, we generate random numbers with Lehmer Random Number Generator (seed: $a=16807$, $\left.\mathrm{z}=172361, \mathrm{~m}=2^{31}-1\right)$ and these numbers $(\bmod 100)$ are 61 , $80,52,61,47,14,12,8,35,55,61,40,96,90,36,49,30$, $34,47,45,92,57,25,97,87,98,26,42,88,82,56,76,55$, $40,80,73,35,45,64,67,91,74,1,47,93,73,29,65,68$, $46,37,50,59,87,83,67,37,32,16,66,97,20,6,53,28$, $35,59,13,2,29,96,84,24,9,11,36,10,7,92,40,18,14$, $58,71,47,40,19,93,40,62,93,97,44,58,6,24,68,54,36$, $25, \ldots$ respectively. Also we take $p$ value randomly such as 0.30 . Consider the parallel version and select pair 7-5 for the first vehicle. The first random number $61 \geq 30$ so we select the pair. We generate random number $80 \geq 30$ for the second pair 10-5 and check the total demand $d_{7}+d_{5}+d_{10}=4500$ less than capacity so we continue to select the pair but pass the pair 8-5 as it surpasses the capacity of the vehicle. The 4th job pair 10-7 is selected. But it is as connected with jobs 7 and 10. Finally, we choose the pair 7-4, generate number $54 \geq 30$ and $d_{7}+d_{5}+d_{10}+d_{4}=5900 \leq 6000$. Hence a new route is created $v_{1}=\left[\begin{array}{llll}4 & 7 & 5 & 10\end{array}\right]$. Then we scan the saving-list and pass to pair which included the same job with first vehicle routes 4-7-5-10. For example; 11-5 is connected with 7-5 (due to job 5) and 9-7 (due to job 7) so we skip it. We choose the disconnected pair 11-8 and generated random number 61 is bigger than $30, d_{11}+d_{8}=3600 \leq 6000$ so $11-8$ is selected for the second vehicle. We continue scanning the saving list till $12-9$, generating random number $47 \geq 30$ but total demand $d_{11}+d_{8}+d_{12}+d_{9}=6500 \geq 6000$ surpasses the capacity so we pass to the disconnected next pair $12-11$. We don't select it because generating number 14 is less than 30 . When similarly continued, pair 9-8 is selected, random number $35 \geq 30$ moreover total demand is $d_{11}+d_{8}+d_{9}=5400$ smaller than the capacity of the second vehicle. Thereby route

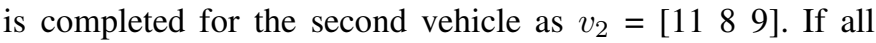
steps applied similarly, we obtain routes for vehicles $v_{3}=[3$ $\left.\begin{array}{lll}2 & 12 & 6\end{array}\right], v_{4}=\left[\begin{array}{ll}1\end{array}\right]$ respectively. So we finish the example.

We now continue with the Binary-CWS-MCS method, which uses the Monte Carlo simulation. Let us explain the method by taking into consideration Example 3. In addition to all algorithmic steps, we apply the simulation (1000 BinaryCWS simulation) for both state of choosing and state of not choosing. At the end of these simulations, we calculate the average score and choose which average score is higher than the other. If we continue to apply each pair in this way, we will create a binary-tree. We give the steps of Binary-CWS-MCS method given in Algorithm 4.

\section{RESUlts}

In this section we compare the results of different pseudorandom generators with different seeds under the CVRP problem. Firstly we take different CVRP problems form TSPLib [16] such as E-13-k4, E-n22-k4, E-n23-k3, E-n30- 
TABLE V

DisTANCE MATRIX
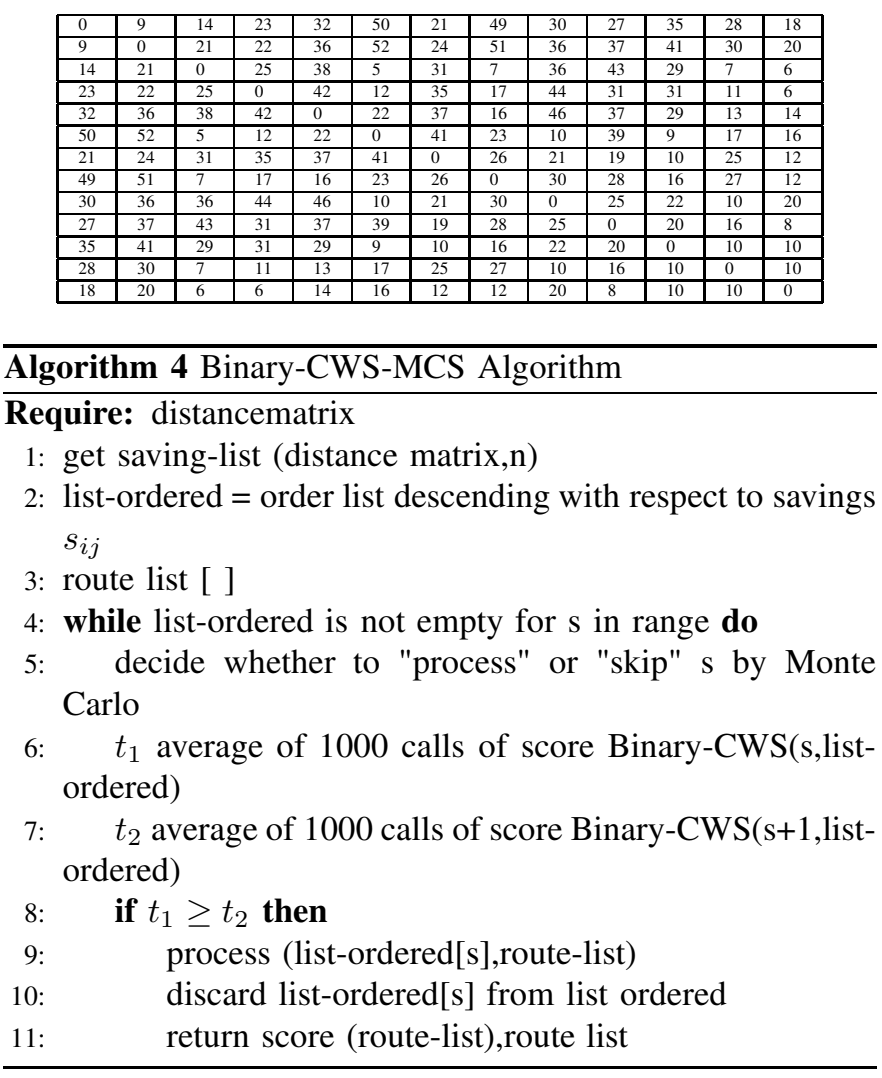

TABLE VI

PARAMETERS OF PSEUDORANDOM NUMBER GENERATORS (PRNG)

\begin{tabular}{|l|l|}
\hline PRNG & Seed \\
\hline LehmerCG & $a: 48271, z: 172361, m: 2^{31}-1$ \\
\hline LCG & {$\left[a: 6364136223846793005, z:[172361], m: 2^{64}\right.$} \\
\hline MRG & {$\left[a: 1071064, z:[135623,172361], m: 2^{31}-19\right.$} \\
\hline ICG & {$\left[a: 197331, z:[172361], m: 2^{31}-1\right.$} \\
\hline EICG & {$\left[a: 197331, z:[172361], m: 2^{48}-59\right.$} \\
\hline
\end{tabular}

k3, E-n31-k7, E-n33-k4, E-n51-k5, E-n76-k7, E-n76-k8, E76k10. For instance E-n51-k5 means that there are 51 jobs and 5 vehicles. Then we calculate the costs of each route by using the pseudorandom numbers generators given in Table VI. We get the shortest route-list as a result for each case. We present the scores in Table VII.

In Table VII, we present the performance comparison of Lehmer Congruential Generator, Linear Congruential Generator, Multiple Recursive Generator, Inversive Congruential Generator, and Explicit Inversive Congruential Generator, whose parameters are given in Table VI. In the light of the table, we see that Lehmer Congruential Generator and Linear Congruential Generator outperform the others. This could be due to their simplicity and good lattice structure as we reduce each random number modulo a number less than 100 to assign it to a job. On the other hand, we see that Explicit Inversive Congruential Generator has dramatically bad results in almost
TABLE VII

PERFORMANCE OF PRNG IN CVRP

\begin{tabular}{|l|l|l|l|l|l|}
\hline VRP/PRNG & LehmerCG & LCG & MRG & ICG & EICG \\
\hline E-n13-k4 & {$[257]$} & {$[257]$} & {$[257]$} & {$[257]$} & {$[257]$} \\
\hline E-n22-k4 & {$[375]$} & {$[376]$} & {$[390]$} & {$[384]$} & {$[381]$} \\
\hline E-n23-k3 & {$[610]$} & {$[583]$} & {$[599]$} & {$[583]$} & {$[615]$} \\
\hline E-n30-k3 & {$[511]$} & {$[512]$} & {$[526]$} & {$[513]$} & {$[508]$} \\
\hline E-n31-k7 & {$[471]$} & {$[470]$} & {$[447]$} & {$[439]$} & {$[508]$} \\
\hline E-n33-k4 & {$[855]$} & {$[860]$} & {$[852]$} & {$[846]$} & {$[861]$} \\
\hline E-n51-k5 & {$[545]$} & {$[558]$} & {$[587]$} & {$[574]$} & {$[566]$} \\
\hline E-n76-k7 & {$[713]$} & {$[733]$} & {$[750]$} & {$[744]$} & {$[749]$} \\
\hline E-n76-k8 & {$[767]$} & {$[767]$} & {$[805]$} & {$[790]$} & {$[809]$} \\
\hline E-n76-k10 & {$[868]$} & {$[889]$} & {$[850]$} & {$[879]$} & {$[889]$} \\
\hline
\end{tabular}

all cases, and so it shouldn't be used for such applications.

It is widely criticized in the literature that the source code of the implemented algorithms needs to be checked by other researchers [20]. Besides, sharing the source code in a public domain opens further improvement and this flourishes the research. In this view, the source code is shared on the web page https://github.com/iedmrc/binary-cws-mcs, which has been implemented according to algorithms in this paper.

\section{ACKNOWLEDGEMENT}

Second and third authors are supported by the Scientific and Technological Research Council of Turkey (TÜBİTAK) under Project No: 116R026.

\section{REFERENCES}

[1] G. Alex , A. A. Juan, J. Faulin, J. de Armas, H. Ramalhinho, "Biased randomization of heuristics using skewed probability distributions: A survey and some applications," Computers \& Industrial Engineering. 15 June 2016.

[2] İ. K. Altınel, and T. Öncan, "A new enhancement of the Clarke and Wright savings heuristic for the capacitated vehicle routing problem," Journal of the Operational Research Society 56.8 (2005): 954-961.

[3] F. Arnold, M. Gendreau, and K. Sörensen, "Efficiently solving very largescale routing problems," Computers \& Operations Research 107 (2019): 32-42.

[4] J. Belloso, A. A. Juan, and J. Faulin, "An iterative biased-randomized heuristic for the fleet size and mix vehicle-routing problem with backhauls," International Transactions in Operational Research 26.1 (2019): 289-301.

[5] L. Calvet, D. Wang, A.A. Juan, and L. Bové, "Solving the multidepot vehicle routing problem with limited depot capacity and stochastic demands," International Transactions in Operational Research 26, no. 2 (2019): 458-484.

[6] G. Clarke and J.W. Wright, "Scheduling of Vehicles from a Central Depot to a Number of Delivery Points," Operations Research, Vol. 12, 1964, pp. 568-581.

[7] P. F. de Córdoba, L. M. García-Raffi, A. Mayado, and J. M. Sanchis, "A real delivery problem dealt with Monte Carlo techniques," Top 8.1 (2000): 57-71.

[8] G. B. Dantzig and J. H. Ramser, "The truck dispatching problem" Management Science, Vol. 6, No. 1 (Oct., 1959), pp. 80-91

[9] T. Doyuran, and B. Çatay, "A robust enhancement to the Clarke-Wright savings algorithm," Journal of the Operational Research Society 62.1 (2011): 223-231.

[10] A.A. Juan, J. Faulín, J. Jorba, D. Riera, D. Masip, and B. Barrios, "On the use of Monte Carlo simulation, cache and splitting techniques to improve the Clarke and Wright savings heuristics," Journal of the Operational Research Society 62, no. 6 (2011): 1085-1097. 
[11] A.A. Juan, S. E. Grasman, J. Caceres-Cruz, and T. Bektaş, "A simheuristic algorithm for the single-period stochastic inventory-routing problem with stock-outs," Simulation Modelling Practice and Theory 46 (2014): 40-52.

[12] G. Laporte, "What You Should Know About the Vehicle Routing Problem," Naval Research Logistics. 2007.

[13] P. L'ecuyer, "Efficient and portable combined random number generators," Communications of the ACM 31.6 (1988): 742-751.

[14] P. L'ecuyer, "Good parameters and implementations for combined multiple recursive random number generators," Operations Research 47.1 (1999): $159-164$

[15] S. K. Park, and K. W. Miller, "Random number generators: good ones are hard to find," Communications of the ACM 31.10 (1988): 1192-1201.

[16] G. Reinelt, "TSPLIB-A traveling salesman problem library," ORSA journal on computing 3.4 (1991): 376-384.

[17] R.Y. Rubinstein, and D.P. Kroese, "Simulation and the Monte Carlo method," Vol. 10. John Wiley \& Sons, 2016.

[18] S, Røpke, "Heuristic and exact algorithms for vehicle routing problems," PhD Thesis, (2006).

[19] B. Saha, K. Suthar, and A. Kumar, "Optimizing Generalized Capacitated Vehicle Routing Problem Using Augmented Savings Algorithm," Computational Intelligence in Data Mining, Springer, Singapore, 2020. 527-541.

[20] K. Sörensen, F. Arnold, and D. Palhazi Cuervo, "A critical analysis of the "improved Clarke and Wright savings algorithm"," International transactions in operational research 26.1 (2019): 54-63.

[21] F. W. Takes, and W. A. Kosters, "Applying Monte Carlo Techniques to the Capacitated Vehicle Routing Problem," 22th Benelux Conference On Artificial Intelligence (BNAIC). 2010.

[22] T. Vidal, G. Laporte and P. Matl, "A concise guide to existing and emerging vehicle routing problem variants," European Journal of Operational Research (2019). 